\title{
Home-based visual field test for glaucoma screening comparison with Humphrey perimeter
}

This article was published in the following Dove Press journal: Clinical Ophthalmology

\section{Stylianos Tsapakis \\ Dimitrios Papaconstantinou \\ Andreas Diagourtas \\ Stylianos Kandarakis \\ Konstantinos Droutsas \\ Konstantinos Andreanos \\ Dimitrios Brouzas}

Ist Department of Ophthalmology, National and Kapodistrian University of Athens, Athens, Greece
Correspondence: Dimitrios Brouzas Ist Department of Ophthalmology, National and Kapodistrian University of Athens, I0 G. Papandreou Str, Byron, Athens I623I, Greece Tel +30210765 2909

$\mathrm{Fax}+302107652909$

Email brouzas@yahoo.com
Purpose: To present a home-based visual field examination method using a PC monitor or virtual reality glasses and evaluate the reliability of the method by comparing the results with those of the Humphrey perimeter, in order to assess the possibility of glaucoma screening through the Internet.

Materials and methods: Software implementing a supra-threshold algorithm for the central $24^{\circ}$ (52 points) of visual field at three threshold levels: 1) $\left.-4 \mathrm{db}, 2\right)-8 \mathrm{db}$, and 3) $-12 \mathrm{db}$, from the age-expected sensitivity was used for the purpose of testing. The software uses the web camera as a "virtual photometer" in order to detect room luminosity and allows self-testing using a computer monitor or virtual reality glasses using an Android smartphone with a 6-inch display. The software includes an expert system to analyze the visual field image and validate the reliability of the results. It also allows the physician to combine the results from two or more tests into a single test in order to achieve higher statistical accuracy of the final result. A total of ten patients, 20 eyes tested $\times 52$ points per eye $=1,040$ visual field test points, were compared point to point to those obtained using the Humphrey perimeter for the same patients, as they appeared randomly and consecutively at the glaucoma department within hours.

Results: Good receiver operating characteristic/area under the curve coefficient was found, ranging from 0.762 to $0.837(P<0.001)$. Sensitivity ranged from 0.637 to 0.942 , and specificity ranged from 0.735 to 0.497 .

Conclusion: The home-based visual field test exhibits a reasonable receiver operating characteristic curve when compared to the Humphrey perimeter, without the need of specialized equipment. The test may be useful for glaucoma screening.

Keywords: glaucoma, screening, internet, computer monitor, android smart-phone, online visual field, virtual reality, teleophthalmology, telemedicine

\section{Introduction}

Glaucoma represents a diverse group of disorders that have common characteristic changes in the optic nerve neuroretinal rim tissue and is a major cause of blindness. It is estimated that half of glaucoma cases are undiagnosed. ${ }^{1}$ The prevalence of glaucoma in the general US population 40 years of age and older is $2.1 \%$, and $\sim 2.4$ million people in the US have undetected and untreated glaucoma. ${ }^{2}$

Visual field testing remains one of the most important exams for determining the stage of glaucoma, the efficacy of medical or surgical treatment, and the prognosis, as well as for assessing the patient's quality of life and his/her ability to perform the activities of daily living. Increasing the frequency of visual field testing leads to earlier detection of glaucoma progression. ${ }^{3}$

The most widely used method to assess visual field deficits in glaucoma is automated perimetry. Despite the several advantages of automated perimetry, there are a 
few disadvantages. Often, it is tiring and difficult for patients to concentrate throughout the test and a large majority of the patients who have visual field test examination belong to the age group over 50. Automated perimeters are objective and accurate devices, but they are also bulky and heavy and are neither portable nor available for home use.

Online visual field testing is interesting for its potential to be used as a low-cost method for glaucoma screening. The potential benefits of an effective online telemedicine system are plentiful, especially in countries with high prevalence of glaucoma or in developing countries, and may save costs to patients and costs to the health care system as a whole.

In the past, various computerized visual field systems were tested, such as: 1) Peristat, 2) Visual Fields Easy (VFE), 3) Testvision.org, and so on. ${ }^{4-7}$

Peristat is an online visual field. It is based on Adobe/Flash technology and runs in a web browser. Flash technology is becoming obsolete. Adobe is planning to remove support for it by the end of 2020 and encourages content creators to migrate to new open formats such as HTML5. Flash technology had security issues. Chrome, Microsoft Edge, and Safari browsers have all been blocking Flash over the past year. In 2010, Steve Jobs banished Adobe Flash from the iPhone. It was too insecure, Jobs wrote, and too resource intensive.

VFE runs on a small smartphone screen (iPhone) which must be held steady by the patient using his/her hands throughout the whole test.

Testvision.org is a test based on ophthalmokinetic perimetry. It is based on Flash technology and has similar limitations to Peristat.

Visual field testing is affected by ambient luminosity. The test has to be performed under specific luminosity conditions in order to achieve standardization in testing conditions, but personal computers/smartphones do not have photometers. None of the previous systems has the ability to check room luminosity.

Visual field testing is also a subjective examination. The patient must be able to understand the testing instructions and must fully cooperate and complete the entire test in order to provide useful information. As a result, visual fields tests have high within-test and between-test variability and it is not rare to have false-positive or false-negative responses. ${ }^{8-10}$ For such reasons, the widespread use of these systems has been limited.

To overcome these issues, a telemedicine visual field test with novel features is presented in this paper. The system 1) uses the web camera as a "virtual photometer" to detect ambient luminosity in order to make sure that the test is performed under standard conditions (dark room); 2) analyzes the visual field image and validates the test results in order to prevent a patient from sending a test to his/her physician if the test was not performed correctly; and 3) allows the physician to combine the results from two or more tests into a single test to achieve higher statistical accuracy of the final result. ${ }^{11}$ The test is based on Microsoft's .NET technology as well as on Google's Android platform.

The purpose of this study was to check the diagnostic ability of the test and calculate its receiver operating characteristic (ROC)/area under the curve (AUC) characteristics as well as the optimal cut-off points when compared with the Humphrey perimeter.

\section{Materials and methods}

To test the reliability of the method, proprietary software implementing a supra-threshold visual field test algorithm at three threshold levels, 1) $-4 \mathrm{db}$ (high level), 2) $-8 \mathrm{db}$ (medium level), and 3) $-12 \mathrm{db}$ (low level), from the ageexpected sensitivity, at the central $24 \%$ (52 points $)$ of visual field was used. ${ }^{12}$

The points are projected using proper trigonometry calculations to compensate for classical perimeter bowl for liquid crystal display (LCD) monitor, so that the stimuli appear on the retina as if they were projected from a classical bowl perimeter. The white/gray stimuli were projected on black background (Figure 1).

The software alternatively allows the use of virtual reality glasses with a 6-inch Android smartphone for visual field testing (Figure 2). If the smartphone uses the same display technology (LCD) as the computer monitor, the results are expected to be comparable.

\section{Display adjustment}

Visual field testing requires specific display luminosity settings. Gamma describes the relationship between the pixel levels and the luminance of the monitor (the light energy it emits). LCD displays show S-shaped curve between the input digital values and the output luminance unlike the conventional cathode ray tube monitor represented by a power function. The relationship between the voltage in an LCD pixel and the light intensity is an S-shaped curve that is nearly linear for the large region between the foot and shoulder of the s-curve. ${ }^{13}$ For this reason, LCD displays are approximately linear devices. Contrast sensitivity decreases by age. ${ }^{14}$ Brightness, contrast, and gamma were adjusted before testing using a step-wedge gray scale. The stepwedge gray scale has 40 distinct shades/steps of gray from 


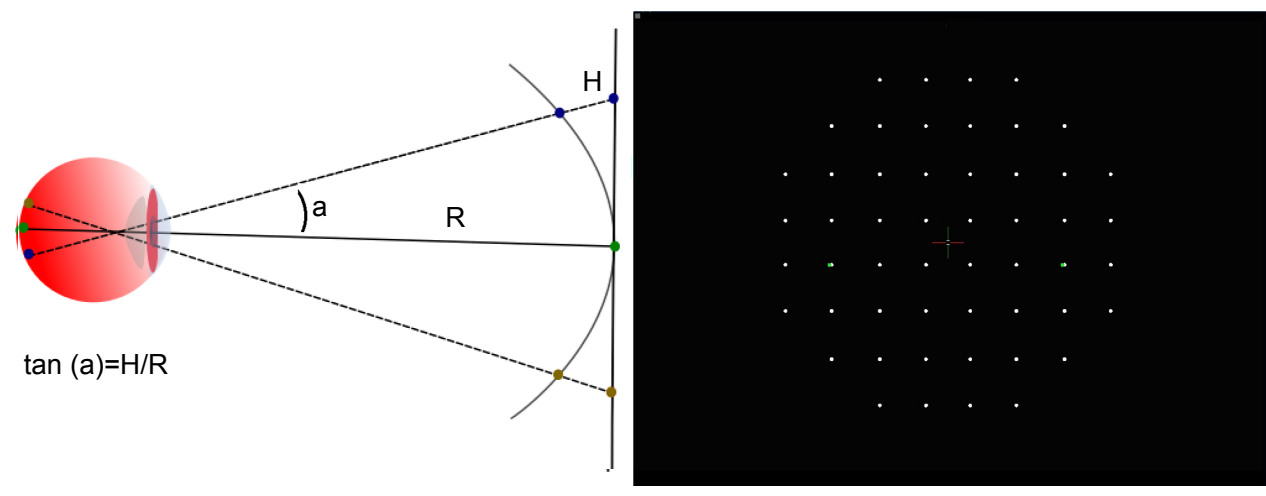

Figure I Trigonometry relation between display and bowl perimeter, 52 points $24^{\circ}$ to be tested.

black to white, with equal differences in brightness between each step. The display was adjusted so that all the shades of gray were distinct and clearly visible. This was about $50 \%$ of the maximum available brightness (Figure 3). ${ }^{15,16}$

When the brightness is properly adjusted, $36-38 \mathrm{~dB}$ should be the approximate upper limit for contrast sensitivity in young observers. ${ }^{12}$ Contrast sensitivity data between different perimeters are similar, albeit not exactly the same. ${ }^{17-20}$

\section{Software features}

1. Supra-threshold strategy, 52 points, central $24^{\circ}$ of visual field (nasally and temporally from the fixation point), at three threshold levels, 1) $-4 \mathrm{db}$ (high level), 2) $-8 \mathrm{db}$ (medium level), and 3) $-12 \mathrm{db}$ (low level), from the age-expected sensitivity. White/gray on black background or black/gray on white background stimuli are available.

2. The software uses the Heijl-Krakau blind spot method to monitor fixation and head position by projecting the stimuli at maximum luminosity at possible blind spot locations until finding the correct response and adjusts stimuli positions and size automatically. ${ }^{21}$

3. Supra-threshold stimuli are used to check for falsenegative results. The software also checks for falsepositive responses by recording positive responses when no response was expected.

4. Variable stimuli presentation rate, adjusted to patient's response time.

5. Stimuli presentation time $250 \mathrm{~ms}$.

6. Statistics. False positive, false negative, fixation losses.

7. Web camera used as virtual "photometer" to detect room luminosity, so that the test is performed under standard conditions (dark room) and records the (red, green, blue) luminosity data in the printout, to provide this information to the doctor.

8. An expert system to analyze the visual field image and detect tests that were not performed correctly.

9. The software allows the doctor to combine the results from two or more tests into a single test to achieve higher statistical accuracy.

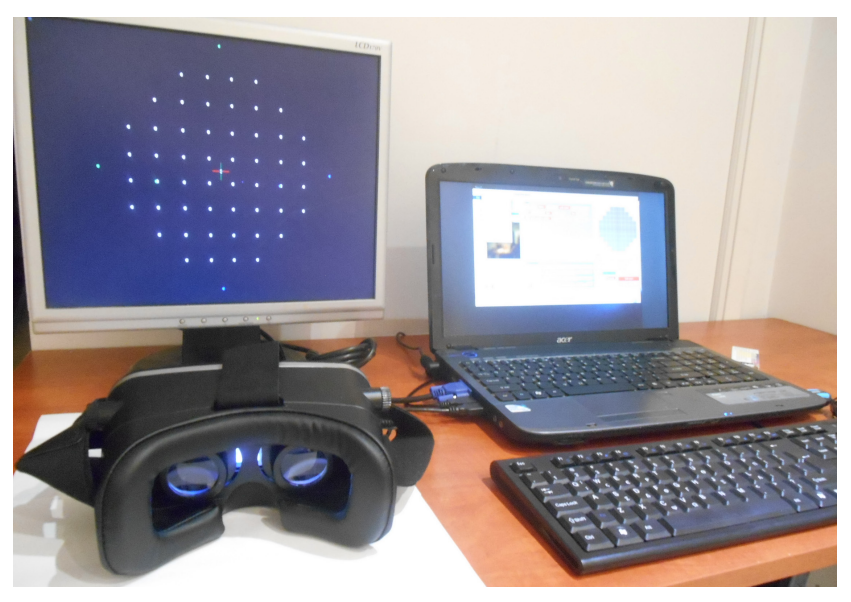

Figure $\mathbf{2}$ Monitor or virtual reality glasses can be used for visual field testing. 


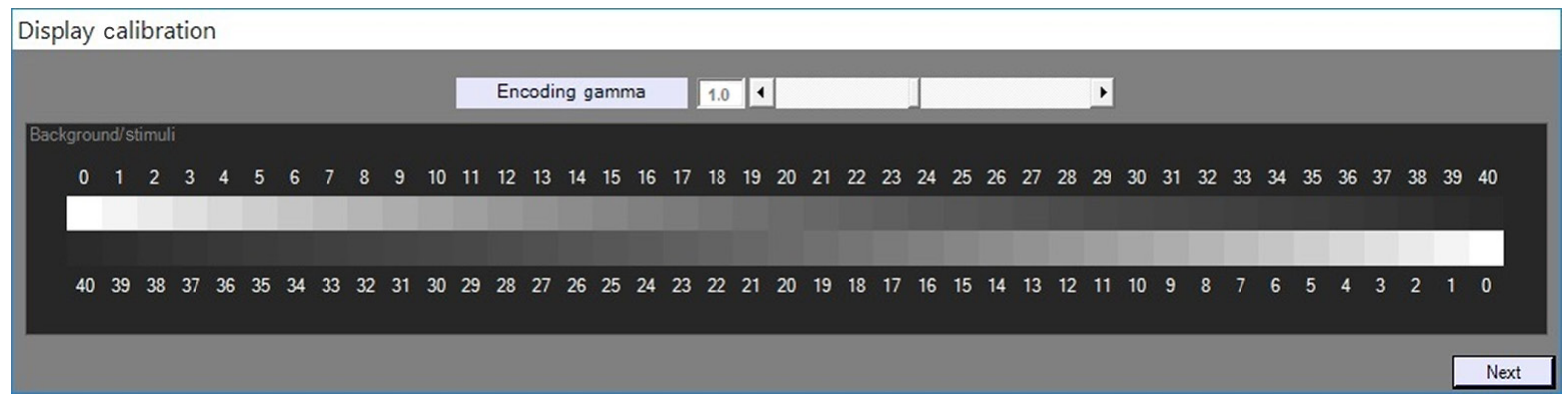

Figure 3 Display - gamma calibration using a gray scale step-wedge.

\section{Examination procedure}

During testing, the user sits comfortably in front of a screen and stares at the central fixation point, while using a mouse to click whenever he/she sees a visual stimulus on the screen. The software has only manual response option. Each eye is tested separately. The users wear their near correction glasses if it is necessary. The eye that is not being tested is covered with the palm of the hand (Figure 4).

All the patients tolerated the test well and they said they found the test simple and easy to perform. The user can test his/her visual field at home and send the results to his/her doctor for evaluation. If the results are not within normal limits, the doctor may advise the patient accordingly, whether or not further examinations are required.

\section{Testing procedure}

To find the right distance from the screen, the following procedure is used. The user closes/covers one eye and stares perpendicularly at the central cross target with the other eye. The user is aware of the blind spot dot, but he/she should not look at it. The user should instead keep his/her eye on the central target. Then, the user slowly moves toward the display, while still staring at the cross target with his/her open eye. At a distance, somewhere around 1-1.2× the height of the display from the computer screen, the blind spot dot will disappear and the area where the blind spot was will be all black. This is the right distance from the computer display. If the user moves closer to the screen or farther away, the blind spot dot will reappear. At just the right distance, the blind spot dot disappears. Peristat online perimetry test uses a similar procedure. Alternatively, the user places his/her eye at a distance $\sim 1-1.2 \times$ the height of the display from the computer screen and stares perpendicularly at the central cross target. The software then locates the blind spot automatically by projecting the stimuli at possible blind spot locations and recording the responses until the user does not see the stimulus/blind spot. Then the locations and sizes of test points are adjusted automatically. If the user is out of limits (too far away or too near from the screen), the blind spot stimuli are visible. In that case, the process fails and a
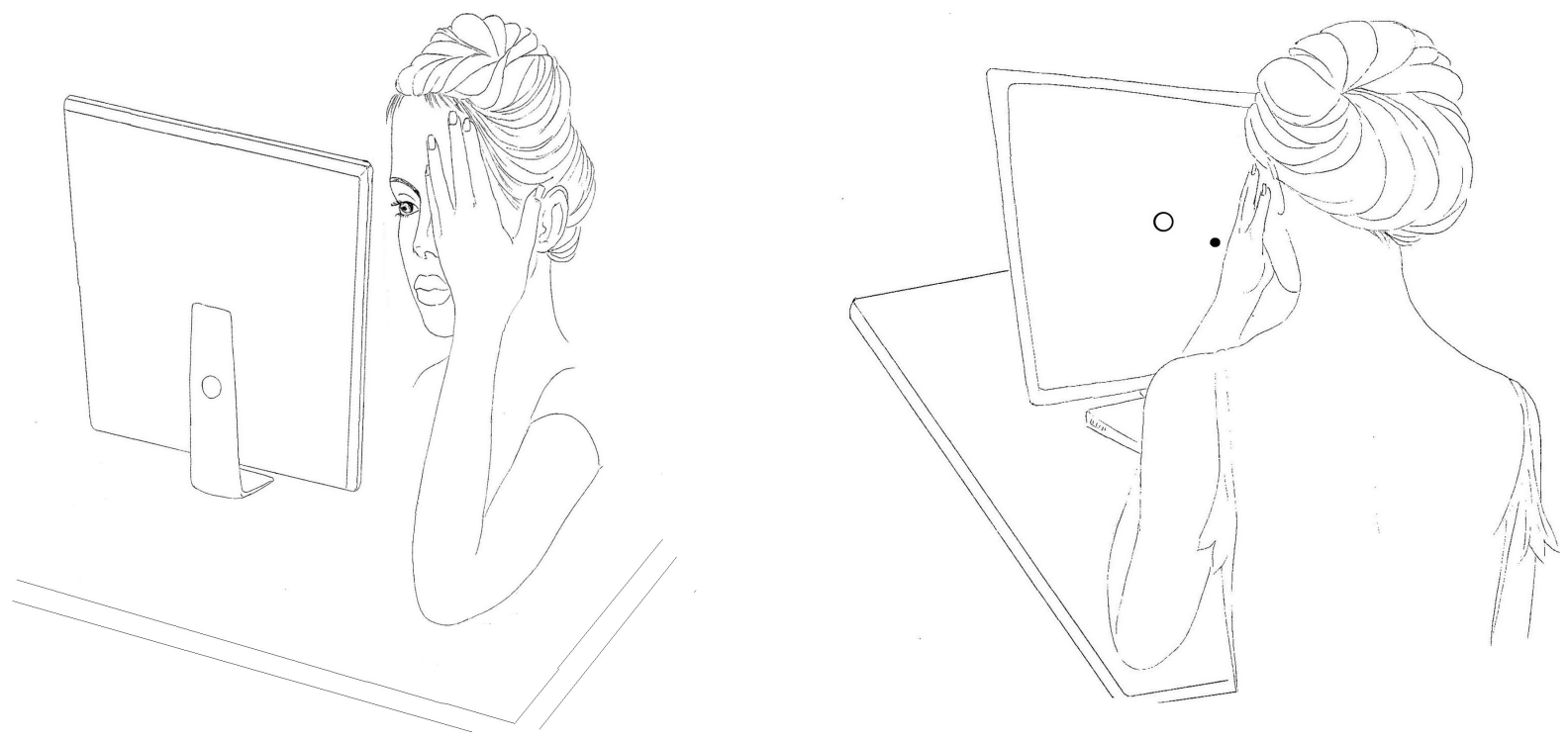

Figure 4 Patient testing in front of a monitor. 
message appears. These methods cannot be applied if there is a scotoma affecting the blind spot or in hemianopic field defects. In that case, all that the patient can do is to place his/her eye at a distance $\sim 1 \times$ the height of the display from the computer screen, stare perpendicularly at the center of the display, and start the test.

The user can select three sensitivity levels either under the guidance of his/her doctor or his/her self-preference.
High sensitivity might detect shallow defects, but has more false-positive findings. Low sensitivity detects deep scotoma only, but has a lower false-positive findings rate. Medium sensitivity gives intermediate results. The system includes eye tracking capability using AForge.NET computer vision and artificial intelligence General Public License library and is able to track the pupil in order to set the central target's location accordingly on the display (Figure 5).

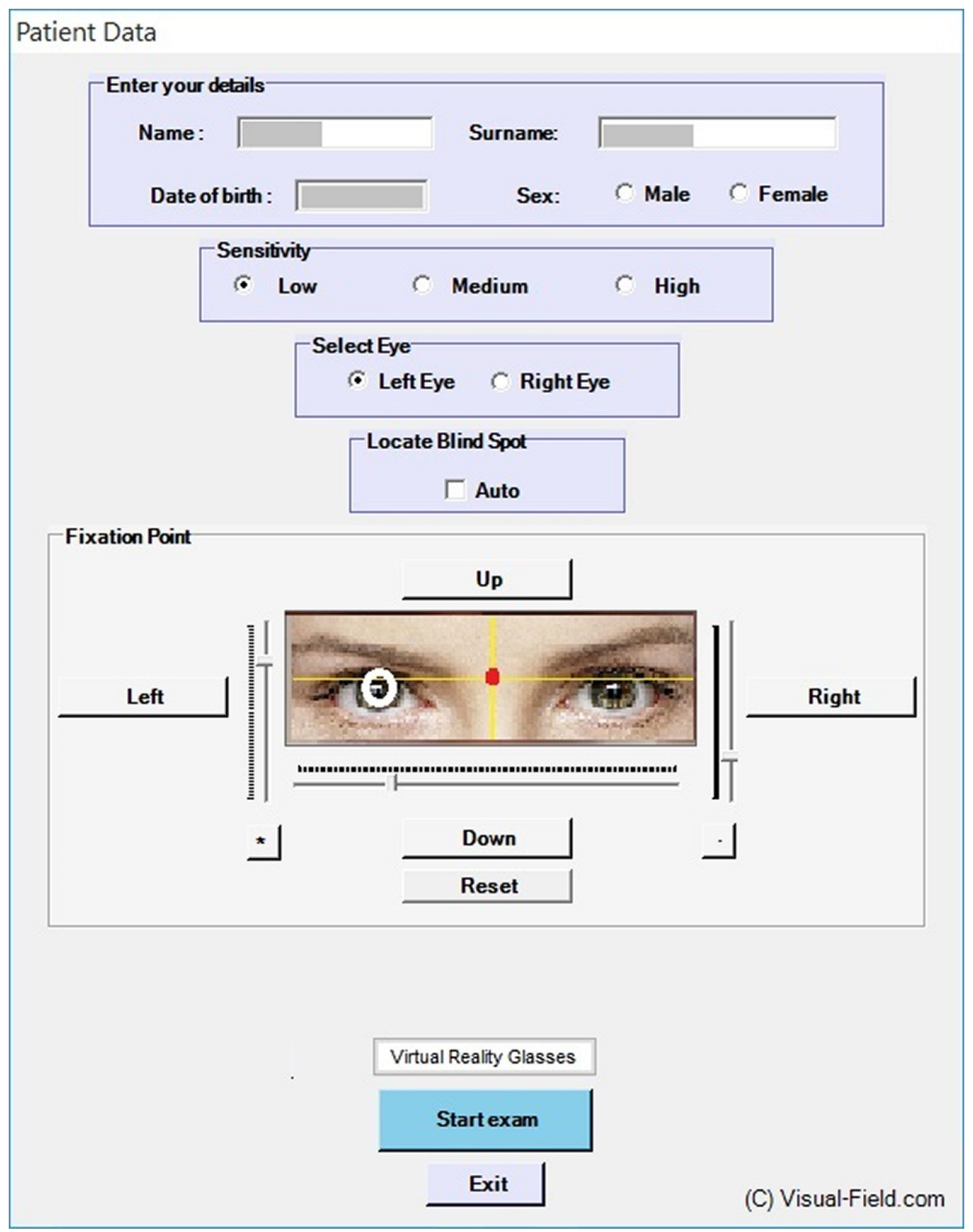

Figure 5 Examination form of the software, eye tracking. 
Test sensitivity is selected at the start of the test. The room should be dark during testing. The web camera is used as a "photometer" to check and record room luminosity before testing starts. The average RGB color values are calculated. RGB color values range from 0 (dark) to 255 .

The tests were performed using a $22^{\prime \prime}$ LCD display, albeit a smaller or bigger monitor could be used because the software can detect the blind spot automatically and calculate the distance between the display and the patient in order to adjust the locations of the projected points as well as their sizes automatically.

During testing, the software projects stimuli at the selected threshold level, at each point tested. If the patient clicks/sees the stimulus, the response is recorded as positive: 1 ; if the patient does not respond, it is recorded as a zero response: 0 . Testing stops when a response, either 1 or 0 , is recorded for each point. The purpose of the test is only to record whether the patient sees or does not see the threshold stimuli. The test is fast (2-3 minutes per eye) because only the selected threshold is tested at each point. The system checks for fixation losses by projecting stimuli at the blind spot. It also checks for false-positive responses and false-negative responses by projecting supra-threshold stimuli at points where the threshold stimulus is found to be visible.

The stimulus presentation rate is variable. The maximum response - waiting time is adjusted to the patient's response time, within limits.

In the results/printout, false-negative test errors are marked in red, while false-positive test errors are marked in green, so that the doctor knows, for example, if a "scotoma" is due to a false-negative test error or a non-scotoma is due to a false-positive test error. Points where false-negative and false-positive errors were recorded are marked in yellow

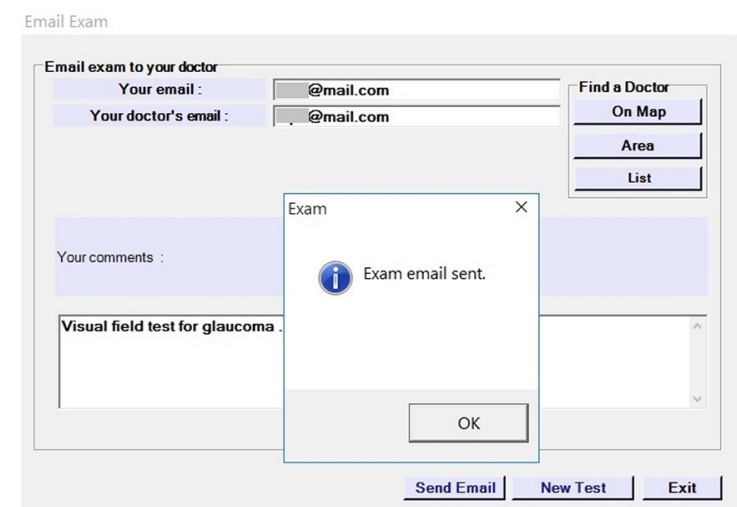

Figure 6 Exam sent by email. color. The exam output is binary (seen/clicked=1, not seen/ not clicked $/$ missed $=0$ ). At the end of the test, the image is analyzed and the results are validated in order to prevent a user from sending a test to his/her doctor, if the test was not performed correctly. A test is considered as "valid" if 1) the blind spot is detected/located at the expected position and 2) the false-positive, false-negative, and fixation losses are all $<25 \%$. If the test is valid, the user can send the test results by email (as an embedded image) to his/her doctor for review (Figure 6).

If the results are not within normal limits, the doctor will advise the patient accordingly whether or not standard automated perimetry and/or further/other examinations are required.

\section{Study participants}

Permission was obtained from the Scientific and Ethical Committee of the General Hospital of Athens "G. Gennimatas" to perform the study. Written informed consent was obtained from all participating patients, and the study adhered to the tenets of the Declaration of Helsinki.

A total of ten patients, 20 eyes tested $\times 52$ points per eye $=1,040$ visual field test points, were compared point to point to those obtained using the Humphrey perimeter for the same patients as they appeared consecutively at the glaucoma department within hours for comparison ${ }^{22,23}$ (Figure 7). All the patients completed the test and were included in the study. The mean age was 67.9 years ( $\mathrm{SD}=12$, ranging from 47 to 81 ).

The results from the visual field screening tests were binary (seen/not seen). ROC curves are widely used in the biomedical sciences and give us the ability to assess the performance of a binary classifier over its entire operating range using a graphical approach. The results were statistically

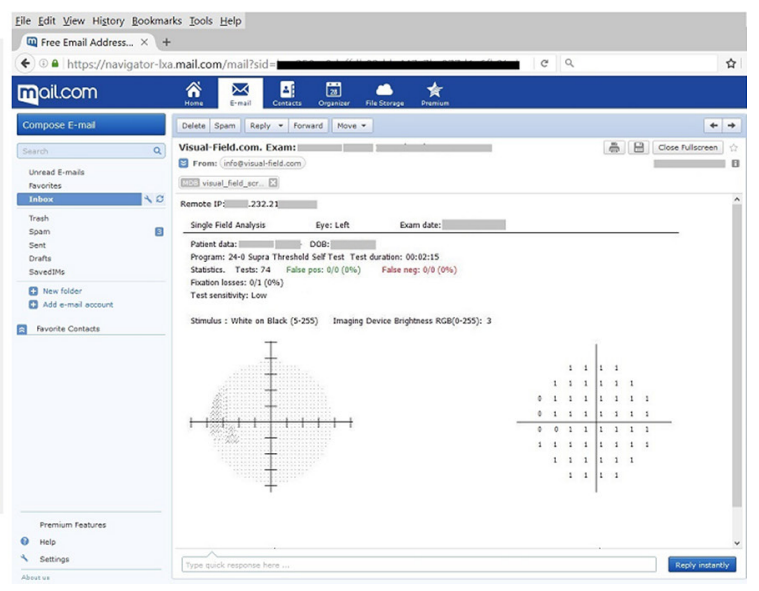




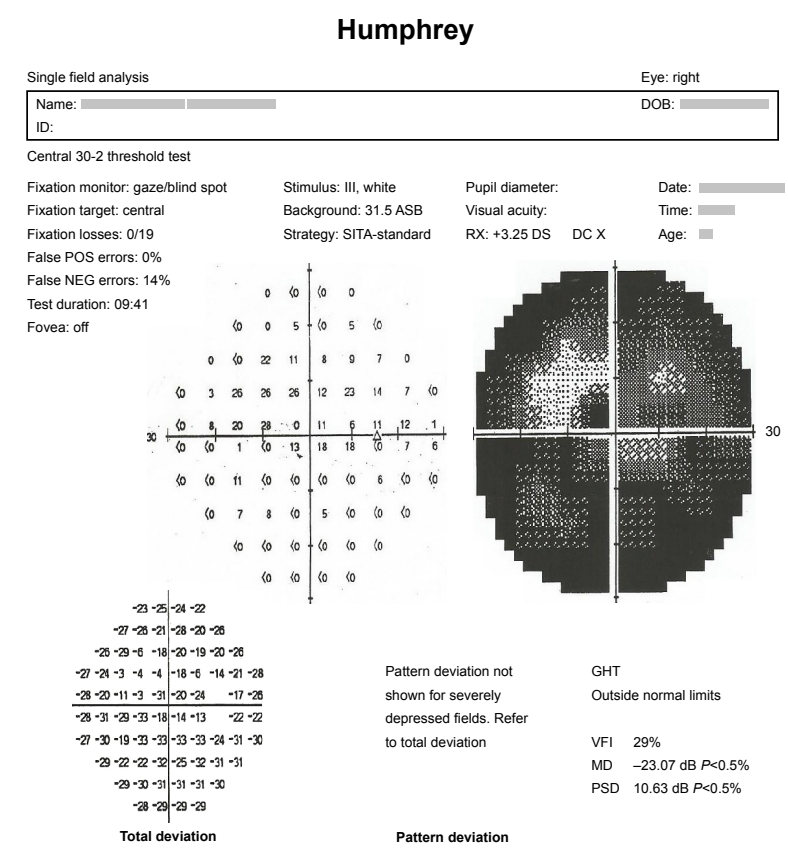

Visual field self test
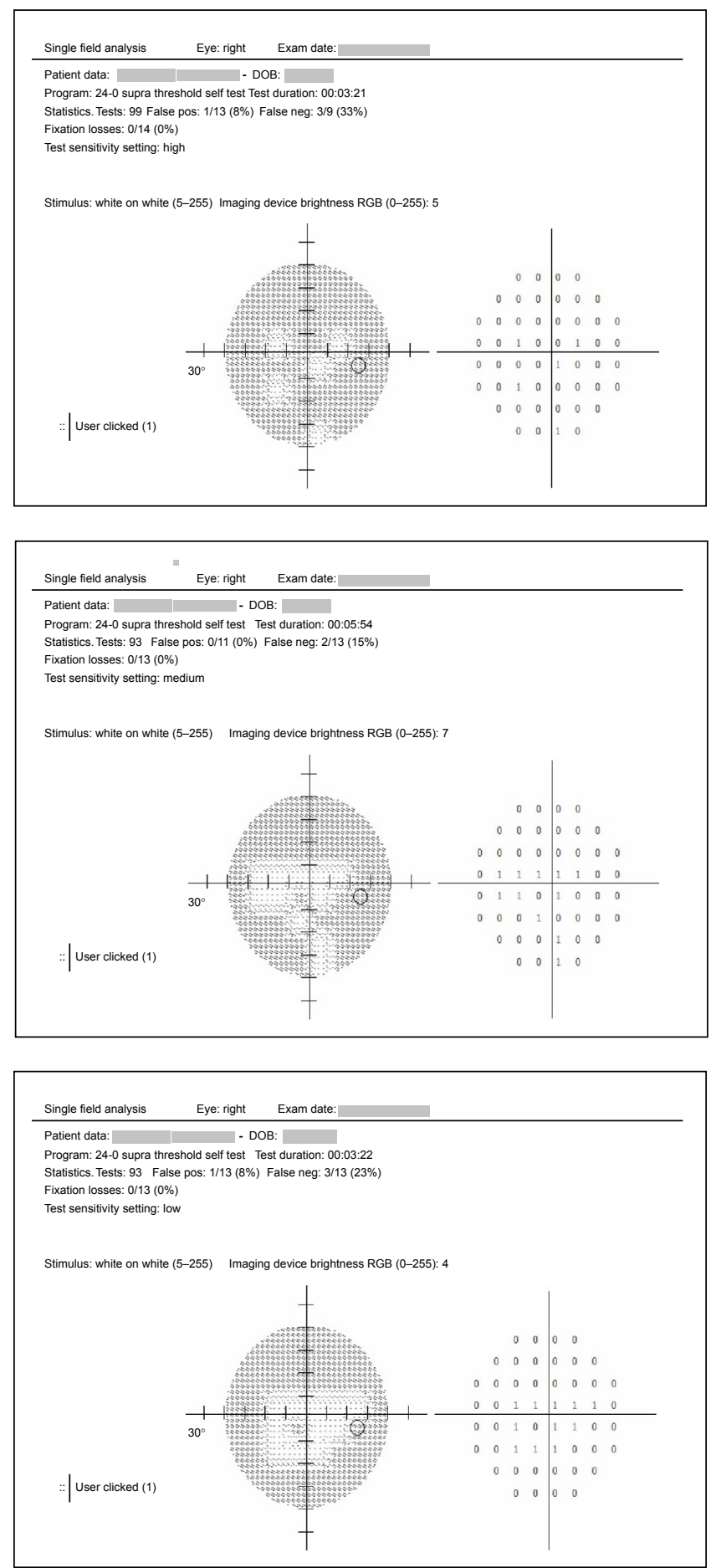

Figure 7 Eye I of 20. Humphrey field analyser test compared with three screening tests at high, medium, and low sensitivity levels (brightest stimulus, $-12 \mathrm{~dB}$ ). Note: As the stimulus gets brighter, the number of positive responses increases.

analyzed and the ROC curves for each threshold level as well as the generalized Youden Index and the optimal cut points were computed using easyROC, an interactive, opensource web tool for ROC curve analysis using R Language Environment. ${ }^{24}$ The easyROC uses the "OptimalCutpoints" software package to determine the cut-off values for diagnostic tests ${ }^{25}$ (Figure 8; Table 1). The reliability indices were also calculated (Table 2). The results were similar, albeit not identical because the algorithms and the technology used were different. 


\section{ROC curves}
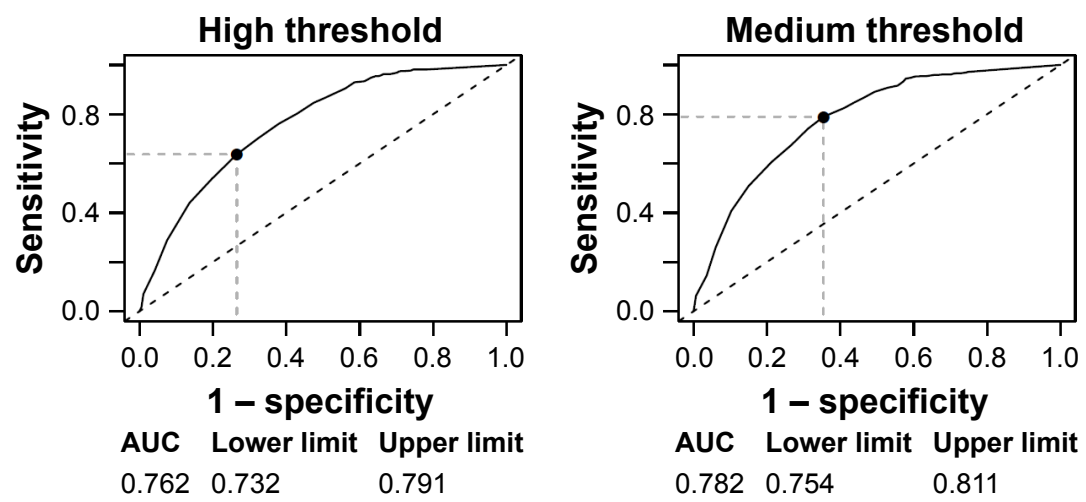

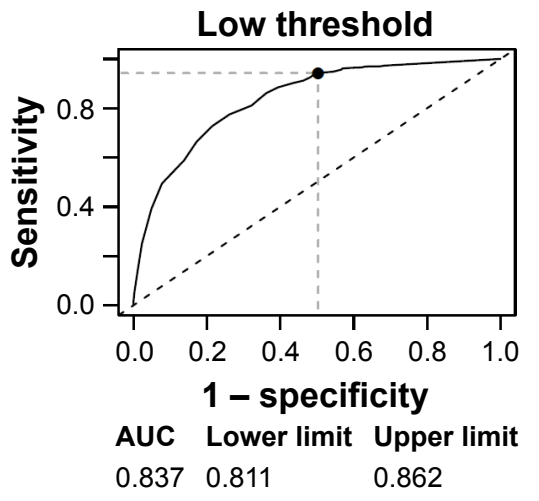

Figure 8 ROC curves for each threshold.

Abbreviations: AUC, area under the curve; ROC, receiver operating characteristic.

\section{Statistical analyses}

The patients were tested successively using a Humphrey perimeter and the telemedicine method. Telemedicine visual field screening tests were $24^{\circ}$ (52 points), while Humphrey field analyser (HFA) tests were $30^{\circ}$ (76) points because the patients were tested with HFA as part of their scheduled/ routine examination and not because of this study. Only the inner common (52) points matching the $24^{\circ}$ central degrees between the HFA and the screening test were compared.

\section{Results}

The results show that the AUC was $0.762(P<0.001)$ for high threshold, $0.782(P<0.001)$ for medium threshold, and $0.837(P<0.001)$ for low threshold.

Table I Threshold data at high, medium, and low sensitivity

\begin{tabular}{|c|c|c|c|}
\hline \multicolumn{4}{|l|}{ High threshold } \\
\hline \multicolumn{4}{|c|}{ Cut-off method: generalized Youden Index optimal cut-off point: 28} \\
\hline & Value & Lower limit & Upper limit \\
\hline Sensitivity & 0.637 & 0.592 & 0.680 \\
\hline Specificity & 0.735 & 0.696 & 0.771 \\
\hline Positive predictive value & 0.675 & 0.632 & 0.715 \\
\hline Negative predictive value & 0.701 & 0.660 & 0.740 \\
\hline Positive likelihood ratio & 2.401 & 2.059 & 2.800 \\
\hline Negative likelihood ratio & 0.494 & 0.435 & 0.562 \\
\hline \multicolumn{4}{|l|}{ Medium threshold } \\
\hline \multicolumn{4}{|c|}{ Cut-off method: generalized Youden Index optimal cut-off point: 25} \\
\hline Sensitivity & 0.790 & 0.755 & 0.822 \\
\hline Specificity & 0.646 & 0.599 & 0.690 \\
\hline Positive predictive value & 0.748 & 0.709 & 0.785 \\
\hline Negative predictive value & 0.697 & 0.654 & 0.738 \\
\hline Positive likelihood ratio & 2.229 & 1.953 & 2.543 \\
\hline Negative likelihood ratio & 0.326 & 0.275 & 0.386 \\
\hline \multicolumn{4}{|l|}{ Low threshold } \\
\hline \multicolumn{4}{|c|}{ Cut-off method: generalized Youden Index optimal cut-off point: I6 } \\
\hline Sensitivity & 0.942 & 0.939 & 0.936 \\
\hline Specificity & 0.497 & 0.503 & 0.509 \\
\hline Positive predictive value & 0.788 & 0.790 & 0.791 \\
\hline Negative predictive value & 0.812 & 0.806 & 0.801 \\
\hline Positive likelihood ratio & 1.874 & 1.889 & 1.906 \\
\hline Negative likelihood ratio & 0.116 & 0.121 & 0.125 \\
\hline
\end{tabular}


Table 2 Reliability indices

\begin{tabular}{|c|c|c|c|c|c|c|c|c|c|c|c|c|}
\hline & \multicolumn{3}{|c|}{ HFA } & \multicolumn{3}{|c|}{ High } & \multicolumn{3}{|c|}{ Medium } & \multicolumn{3}{|c|}{ Low } \\
\hline & $\mathbf{F L}$ & FP & FN & FL & FP & FN & FL & FP & FN & FL & FP & FN \\
\hline $\begin{array}{l}\text { Mean } \\
\text { SD }\end{array}$ & $\begin{array}{l}16 \\
26\end{array}$ & $\begin{array}{l}1.5 \\
2.1\end{array}$ & $\begin{array}{l}7.4 \\
12\end{array}$ & $\begin{array}{l}16 \\
26\end{array}$ & $\begin{array}{l}17 \\
13\end{array}$ & $\begin{array}{l}20 \\
23\end{array}$ & $\begin{array}{l}22 \\
32\end{array}$ & $\begin{array}{l}13 \\
12\end{array}$ & $\begin{array}{l}10 \\
19\end{array}$ & $\begin{array}{l}23 \\
29\end{array}$ & $\begin{array}{l}17 \\
14\end{array}$ & $\begin{array}{l}12 \\
16\end{array}$ \\
\hline
\end{tabular}

Abbreviations: FL, fixation losses; FN, false negative; FP, false positive; HFA, Humphrey field analyser.

In summary, we see that as the threshold tested gets lower, the sensitivity increases while the specificity decreases and vice versa. The optimal cut-off point for Humphrey data was $28 \mathrm{~dB}$ for the high threshold, $25 \mathrm{~dB}$ for the medium threshold, and $16 \mathrm{~dB}$ for the low threshold.

Black/gray stimuli on white background were also tested with similar results, but they are not included in this paper.

In practice, when a single test is used repeatedly in routine screening, the same screening threshold is typically used at each screening visit. One possible alternative is to adjust the threshold at successive visits according to individualspecific characteristics. ${ }^{26}$ The test results can be combined in series or in parallel. ${ }^{27}$ Parallel testing is recommended for ruling in diagnoses, while series testing is recommended for ruling out diagnoses. ${ }^{28}$ The software allows the user to select and combine the results from two or more tests into a single test. The sum of positive responses at each point merged is shown.

\section{Discussion}

Telemedicine visual field screening testing has many similarities to classical bowl perimetry, but there are some differences due to the hardware used.

We presented a visual field test with novel features and evaluated the reliability of the method by comparing the results with those of Humphrey perimeter.

The purpose of the test is to help glaucoma patients become aware of their problem, albeit other disorders that affect the visual field might be detected as well.

The advantages of our system are that it uses the web camera as a "photometer" and validates the reliability of the results when the test is completed. This system also allows the patient to send the results to his/her doctor by email and allows the doctor to combine the results from two or more tests into a single test to achieve higher statistical accuracy.

The test is simple, easy, and fast and does not require specialized equipment. It only takes $2-3$ minutes per eye and can be repeated as many times as needed. The patients were asked at the end of the test and they reported that it was easy and simple.
The test implements a supra-threshold visual field test algorithm at three sensitivity levels. It is intended to indicate whether there are findings in the visual field that might require further examinations. Standard automated perimetry test should be used to quantify the defects. The test is not intended to monitor the progression of diagnosed cases, but to be used as a screening test.

The optimal cut-off points as well as the ROC/AUC characteristics of this test were calculated when comparing the results with those obtained from the Humphrey perimeter for the same group of patients. ROC analysis is a widely used method for evaluating the accuracy of medical diagnostic systems. The most desirable property of ROC analysis is that the accuracy indices derived from this technique are not distorted by fluctuations caused by the use of arbitrarily chosen decision criteria or cut-offs. In other words, the indices of accuracy are not influenced by the decision criterion. Using this as a measure of a diagnostic performance, one can compare individual tests or judge whether the various combination of tests can improve diagnostic accuracy. ${ }^{29}$ Good ROC/AUC coefficient was found, ranging from 0.762 to $0.837(P<0.001)$. Sensitivity ranged from 0.63 to 0.94 , and specificity ranged from 0.73 to 0.49 .

Other systems have found similar results. Peristat online perimetry had comparable ROC curves and Spearman's rank correlation coefficient ranging from 0.55 to 0.77 (all $P<0.001)$ when compared with the Humphrey perimeter. ${ }^{30}$ VFE, which is an iPad application for visual field testing, had ROC/AUC ranging from 0.687 to $0.784 . .^{31,32}$

The prevalence of glaucoma increases with age. It is most common in adults in their $70 \mathrm{~s}$ and $80 \mathrm{~s}$. The main limitation of this test is that many older people never learned to use computers. Barriers may include physical and mental limitations. As with any other computer system, older people may still need a little help and support by younger people, family, or professionals who care for older adults.

\section{Conclusion}

The ROC characteristics of this low-cost test show it is reliable at least when compared with the Humphrey perimeter and does not require specialized equipment. The test may be useful for home-based glaucoma screening. 


\section{Data sharing statement}

The datasets generated during and/or analyzed during the current study are available from the corresponding author on reasonable request. Non-profit institutions can freely avail the software by contacting the corresponding author or by sending an email to info@visual-field.com.

\section{Ethics approval and consent to participate}

Permission was obtained from the Scientific and Ethical Committee of the General Hospital of Athens "G. Gennimatas" to perform the study. Written informed consent was obtained from all participating patients, and the study adhered to the tenets of the Declaration of Helsinki.

\section{Disclosure}

The authors report no conflicts of interest in this work.

\section{References}

1. Shaikh Y, Yu F, Coleman AL. Burden of undetected and untreated glaucoma in the United States. Am J Ophthalmol. 2014;158(6): $1121-1129$

2. Gupta P, Zhao D, Guallar E, Ko F, Boland MV, Friedman DS. Prevalence of glaucoma in the United States: the 2005-2008 National Health and Nutrition Examination Survey. Invest Ophthalmol Vis Sci. 2016; 57(6):2905-2913.

3. Nouri-Mahdavi K, Zarei R, Caprioli J. Influence of visual field testing frequency on detection of glaucoma progression with trend analyses. Arch Ophthalmol. 2011;129(12):1521-1527.

4. Bruun-Jensen J. Visual field screening with a laptop computer system. Optometry. 2011;82(9):519-527.

5. Ianchulev T, Pham P, Makarov V, Francis B, Minckler D. Peristat: a computer-based perimetry self-test for cost-effective population screening of glaucoma. Curr Eye Res. 2005;30(1):1-6.

6. Vingrys AJ, Healey JK, Liew S, et al. Validation of a tablet as a tangent perimeter. Transl Vis Sci Technol. 2016;5(4):3.

7. Damato B, Groenewald C. Multifixation campimetry on line: a perimeter for the detection of visual field loss using the internet. Br JOphthalmol. 2003;87(10):1296-1298.

8. Wall M, Kutzko KE, Chauhan BC. Variability in patients with glaucomatous visual field damage is reduced using size V stimuli. Invest Ophthalmol Vis Sci. 1997;38(2):426-435.

9. Anderson AJ. Significant glaucomatous visual field progression in the first two years: what does it mean? Transl Vis Sci Technol. 2016; $5(6): 1$.

10. Chauhan BC, Garway-Heath DF, Goñi FJ, et al. Practical recommendations for measuring rates of visual field change in glaucoma. $\mathrm{Br} \mathrm{J}$ Ophthalmol. 2008;92(4):569-573.

Clinical Ophthalmology

\section{Publish your work in this journal}

Clinical Ophthalmology is an international, peer-reviewed journal covering all subspecialties within ophthalmology. Key topics include: Optometry; Visual science; Pharmacology and drug therapy in eye diseases; Basic Sciences; Primary and Secondary eye care; Patient Safety and Quality of Care Improvements. This journal is indexed on Submit your manuscript here: http://www.dovepress.com/clinical-ophthalmology-journal
11. Christodouleas DC, Nemiroski A, Kumar AA, Whitesides GM. Broadly available imaging devices enable high-quality low-cost photometry. Anal Chem. 2015;87(18):9170-9178.

12. Anderson AJ, Johnson CA, Fingeret M, et al. Characteristics of the normative database for the Humphrey matrix perimeter. Invest Ophthalmol Vis Sci. 2005;46(4):1540-1548.

13. To L, Woods RL, Goldstein RB, Peli E. Psychophysical contrast calibration. Vision Res. 2013;90:15-24.

14. Bowling B. Kanski's Clinical Ophthalmology: a systematic approach. 8th ed. United Kingdom: Elsevier; 2016:209.

15. Xiao K, Fu C, Karatzas D, Wuerger S. Visual gamma correction for LCD displays. Displays. 2011;32(1):17-23.

16. Colombo E, Derrington A. Visual calibration of CRT monitors. Displays. 2001;22(3):87-95.

17. Anderson DR, Feuer WJ, Alward WL, Skuta GL. Threshold equivalence between perimeters. Am J Ophthalmol. 1989;107(5):493-505.

18. Wong AY, Dodge RM, Remington LA. Comparing threshold visual fields between the Dicon TKS 4000 automated perimeter and the Humphrey Field Analyzer. J Am Optom Assoc. 1995;66(11):706-711.

19. Lewis RA, Johnson CA, Keltner JL, Labermeier PK. Variability of quantitative automated perimetry in normal observers. Ophthalmology. 1986;93(7):878-881.

20. Brenton RS, Argus WA. Fluctuations on the Humphrey and Octopus perimeters. Invest Ophthalmol Vis Sci. 1987;28(5):767-771.

21. Heijl A, Krakau CE. A note of fixation during perimetry. Acta Ophthalmol. 1977;55(5):854-861.

22. Bloch DA. Comparing two diagnostic tests against the same "gold standard" in the same sample. Biometrics. 1997;53(1):73-85.

23. Hanley JA, Mcneil BJ. A method of comparing the areas under receiver operating characteristic curves derived from the same cases. Radiology. 1983;148(3):839-843.

24. Goksuluk D, Korkmaz S, Zararsiz G, Karaağaoğlu AE. easyROC: an interactive web-tool for ROC curve analysis using R language environment. $R$ J. 2016;8(2):213-230.

25. López-Ratón M, Rodríguez-Álvarez MX, Suárez CC, Sampedro FG, Cadarso Suarez C, Gude Sampedro F. OptimalCutpoints: an R package for selecting optimal cutpoints in diagnostic tests. J Stat Softw. 2014; 61(8):1-36

26. Thompson ML. Assessing the diagnostic accuracy of a sequence of tests. Biostatistics. 2003;4(3):341-351.

27. Lewis G, Sheringham J, Bernal JL, Crayford T. Mastering Public Health: A Postgraduate Guide to Examinations and Revalidation. 2nd ed. Boca Raton, FL: CRC Press/Taylor \& Francis Group; 2014:190-191.

28. Zou KH, Bhagwat JG, Carrino JA. Statistical combination schemes of repeated diagnostic test data. Acad Radiol. 2006;13(5):566-572.

29. Hajian-Tilaki K. Receiver operating characteristic (ROC) curve analysis for medical diagnostic test evaluation. Caspian J Intern Med. 2013;4(2):627-635.

30. Lowry EA, Hou J, Hennein L, et al. Comparison of Peristat online perimetry with the Humphrey perimetry in a clinic-based setting. Transl Vis Sci Technol. 2016;5(4):4.

31. Kong YX, He M, Crowston JG, Vingrys AJ. A comparison of perimetric results from a tablet perimeter and Humphrey field analyzer in glaucoma patients. Transl Vis Sci Technol. 2016;5(6):2.

32. Johnson CA, Thapa S, George Kong YX, Robin AL. Performance of an iPad application to detect moderate and advanced visual field loss in Nepal. Am J Ophthalmol. 2017;182:147-154.

\section{Dovepress}

PubMed Central and CAS, and is the official journal of The Society of Clinical Ophthalmology (SCO). The manuscript management system is completely online and includes a very quick and fair peer-review system, which is all easy to use. Visit http://www.dovepress.com/ testimonials.php to read real quotes from published authors. 